\title{
Sorafenib Therapy for BCLC Stage B/C Hepatocellular Carcinoma; Clinical Outcome and Safety in Aged Patients: A Multicenter Study in Japan
}

\author{
Hiroki Nishikawa ${ }^{1 \bowtie *}$, Haruhiko Takeda ${ }^{1 *}$, Kaoru Tsuchiya ${ }^{2}$, Kouji Joko $^{3}$, Chikara Ogawa 4 , Hiroyoshi \\ Taniguchi ${ }^{5}$, Etsuro Orito ${ }^{6}$, Yasushi Uchida7, Yukio Osaki ${ }^{1}$, Namiki Izumi²; Japanese Red Cross Liver Study \\ Group \\ 1. Department of Gastroenterology and Hepatology, Osaka Red Cross Hospital; \\ 2. Department of Gastroenterology and Hepatology, Musashino Red Cross Hospital; \\ 3. Center for Liver-Biliary-Pancreatic Diseases, Matsuyama Red Cross Hospital; \\ 4. Department of Gastroenterology, Takamatsu Red Cross Hospital; \\ 5. Department of Gastroenterology, Japanese Red Cross Medical Center; \\ 6. Department of Gastroenterology and Hepatology Nagoya Daini Red Cross Hospital; \\ 7. Department of Gastroenterology, Matsue Red Cross Hospital. \\ * Hiroki Nishikawa and Haruhiko Takeda equally contributed to this work. \\ $\triangle$ Corresponding author: Hiroki Nishikawa, Department of Gastroenterology and Hepatology, Osaka Red Cross Hospital, 5-30 \\ Fudegasaki-cho, Tennoji-ku, Osaka 543-0027, Japan. Tel: +81-6-6774-5111; Fax: +81-6-6774-5131 E-mail: h-nishikawa@osaka-med.jrc.or.jp.
}

(c) Ivyspring International Publisher. This is an open-access article distributed under the terms of the Creative Commons License (http://creativecommons.org/ licenses/by-nc-nd/3.0/). Reproduction is permitted for personal, noncommercial use, provided that the article is in whole, unmodified, and properly cited.

Received: 2014.03.31; Accepted: 2014.05.II; Published: 2014.06.07

\begin{abstract}
Background and aims: We aimed to compare clinical outcomes and safety after sorafenib therapy between patients with Barcelona Clinic Liver Cancer (BCLC) stage B or C hepatocellular carcinoma (HCC) aged $\geq 75$ years (aged group, $n=179$ ) and those with $B C L C$ stage $B$ or $C$ HCC aged $<75$ years (control group, $\mathrm{n}=279$ ).

Patients and methods: We compared overall survival (OS), progression free survival (PFS), best treatment response and sorafenib related serious adverse events (SAEs) of grade 3 or more in the two groups. Furthermore, for reducing the selection bias, we compared clinical outcome of these two groups using propensity score matching analysis.

Results: The median OS and PFS intervals were 9.7 and 3.8 months in the aged group and 8.2 and 3.3 months in the control group ( $P=0.64$ I for OS and $P=0.068$ for PFS). Disease control rates were $49.2 \%(88 / 179)$ in the aged group and $49.1 \%$ (137/279) in the control group $(P>0.999)$. Objective response rates were $15.1 \%(27 / 179)$ in the aged group and $14.3 \%(40 / 279)$ in the control group $(P=0.892)$. Treatment related SAEs of grade 3 or more were observed in 5 I patients $(28.5 \%)$ in the aged group and in 69 patients $(24.7 \%)$ in the control group $(P=0.385)$. In the propensity score matched cohort (132 pairs), no significant difference in the two groups was observed in terms of OS $(P=0.898)$ and PFS $(P=0.407)$.

Conclusion: In BCLC stage B or C HCC patients treated with sorafenib, life expectancy, disease progression, treatment efficacy and SAEs are unaffected by age over 75 years.
\end{abstract}

Key words: Hepatocellular carcinoma, Sorafenib, Aged patients, Clinical outcome, Safety.

\section{Introduction}

Hepatocellular carcinoma (HCC) ranks fifth among the most prevalent deadly cancers in the world, and is the third most common cause of cancer related death. [1-4] The incidence of cancer has been reported to increase markedly with age, with $>60 \%$ of all cancers developing in patients aged 65 years or more. [5] The risk of HCC development is known to be age dependent. [6] Thus, there will be an increasing 
number of elderly HCC patients in the coming years owing to the increased longevity of the population. The current established HCC therapy includes surgical resection, liver transplantation, transcatheter arterial chemoembolization (TACE), ablative therapies such as radiofrequency ablation (RFA) or percutaneous ethanol injection (PEI) and molecular targeted drug (sorafenib). [3]

Sorafenib, a multi-kinase inhibitor that blocks tumor growth and cell proliferation, was the first systemic chemotherapeutic drug found to improve the survival time of patients with advanced HCC both in the SHARP trial and the Asian Pacific trial. [7-9] Sorafenib has thus opened a novel era for the treatment of advanced HCC. In general, sorafenib therapy is indicated for patients with Barcelona Clinic Liver Cancer (BCLC) stage B HCC who are refractory to or had contraindications to locoregional therapies or for patients with BCLC stage C HCC. [7, 9]

As compared with younger patients, elderly patients generally have more comorbid diseases. Furthermore, in the management of elderly patients with advanced cancer, systemic chemotherapy is frequently either modified or withheld for fear of potential toxicities to chemotherapy. [10] To our knowledge, there have been few reports on clinical outcomes and safety in elderly HCC patients treated with sorafenib, although there have been several reports regarding patients treated with other therapies such as surgical resection, RFA, PEI and TACE. [11-32] Thus, there is urgent need for investigation of clinical outcomes and safety in elderly patients with HCC treated with sorafenib and this is a relevant topic for clinicians.

We have conducted a multicenter study of sorafenib therapy for HCC in Japanese Red Cross Liver Study Group. The aims of the present study were to evaluate clinical outcomes and safety after sorafenib therapy in BCLC stage B or C HCC patients aged $\geq 75$ years as compared with BCLC stage B or C HCC patients aged $<75$ years. Furthermore, for reducing the selection bias, we compared clinical outcome of these two groups using propensity score matching analysis.

\section{Patients and Methods}

\section{Patients}

A total of 465 patients with unresectable HCC in the Japanese Red Cross Liver Study Group were treated with sorafenib between June 2008 and August 2013.

Our indications for sorafenib therapy were as follows: (1) Child-Pugh classification of A or B, (2) Eastern Cooperative Oncology Group (ECOG) performance status (PS) of 0, 1 or 2 (3) unresectable HCC determined by dynamic computed tomography (CT) scan and/or magnetic resonance imaging (MRI), (4) presence of extrahepatic metastases, (5) refractory to previous HCC therapies such as TACE and other locoregional therapies, (6) unsuitability for TACE for anatomical reasons, or (7) with vascular invasion such as portal vein tumor thrombus. However, in cases with Child-Pugh $C$ in whom no other effective therapy could be adoptable, sorafenib was given according to decision by each attending physician after full explanation for sorafenib therapy. Of 465 patients, 7 patients had BCLC stage A or D. Thus, a total of 458 patients were analysed in the current analysis. They included 179 patients aged 75 years old or more (the aged group) and 279 patients aged less than 75 years old (the control group). We chose the cut off age of 75 years considering the aging population of HCC patients in our country. $[12,15-19,29-32]$ In addition, in our country, patients aged $\geq 75$ years are covered by a health insurance system which is different from that for patients aged $<75$ years. All patients analysed had at least one dose of sorafenib.

We compared the clinical outcomes and safety including overall survival (OS), progression-free survival (PFS), best response rate during follow-up period and SAEs of grade 3 or more between these two groups after sorafenib therapy. Prior to sorafenib therapy, written informed consent was obtained from all patients. The current study comprised a retrospective analysis of patient records and all treatments were conducted in an open-label manner. The ethics committees of all facilities that participated in this study approved the current study protocol and this study protocol complied with all of the provisions of the Declaration of Helsinki.

\section{HCC diagnosis}

HCC was diagnosed using abdominal ultrasound, dynamic computed tomography (CT) scans (hyperattenuation during the arterial phase in all or some part of the tumor and hypoattenuation in the portal-venous phase) and/or MRI. [33] In some patients, CT hepatic angiography (CTHA) and CT arterio-portography (CTAP) were performed to confirm HCC diagnosis. All patients were confirmed ineligible for surgery or locoregional therapies radiologically. In 90 patients $(19.7 \%)$ out of 458 patients analysed in this study, percutaneous tumor biopsy was performed. They included Edmondson grade I (Ed. I) HCC in 29 patients, Ed. II HCC in 37, Ed. III HCC in 23 and mixed type HCC in one.

\section{Sorafenib therapy}

The recommended initial dose of sorafenib for HCC is 400 mg twice a day. [7-9] Nevertheless, ac- 
cording to the phase-I study by Miller et al., patients with insufficient liver function or renal function (serum total bilirubin $>1.5 \mathrm{mg} / \mathrm{dL}$, serum albumin $<2.5$ $\mathrm{g} / \mathrm{dL}$ and/or creatinine clearance $<40 \mathrm{mg} / \mathrm{mL}$ ) are recommended to receive a reduced dose of sorafenib. [34] Studies in several countries have reported SAEs in several individuals with advanced HCC given an initial dose of sorafenib of $800 \mathrm{mg} /$ day, which led to treatment discontinuation. Moreover, in Japan, the proportion of the population with a body mass index (BMI) of $\geq 30 \mathrm{~kg} / \mathrm{m}^{2}$ has been reported to be lower than that in western countries and the recommended initial dose of sorafenib in elderly patients with advanced HCC is not well established because of paucity of available data. [35-37] Taking this information into consideration, the initial dose of sorafenib was determined according to factors such as body weight (BW), BMI, age, comorbid diseases, PS and liver function. Hence, the initial sorafenib dose in this study ranged from $200-800 \mathrm{mg} /$ day. In cases of sorafenib related SAEs of grade 3 or more as defined by the National Cancer Institute Common Terminology Criteria for Adverse Events (CTCAE), sorafenib dose was reduced by 200 to $400 \mathrm{mg} /$ day at the discretion of attending physicians. Temporary treatment interruptions were also allowed. Sorafenib treatment continued until disease progression, unacceptable drug-related toxicity, or the patient's wish to discontinue treatment. Even if sorafenib therapy was discontinued, in patients who were potentially tolerable for other anti-cancer therapies, post-sorafenib therapies such as other systemic chemotherapies, TACE, transcathether arterial infusion chemotherapy and several ongoing clinical trials as second-line chemotherapy were considered.

\section{Assessments of treatment efficacy and follow up}

Best treatment efficacy of sorafenib during treatment was assessed in accordance with the modified Response Evaluation Criteria in Solid Tumors for Hepatocellular Carcinoma (mRECIST) criteria and/or tumor marker levels. [38] The treatment efficacy was classified as: (1) complete response (CR), (2) partial response (PR), (3) stable disease (SD) and progressive disease (PD). CR was defined as disappearance of any arterial enhancement within all target tumors. PR was defined as $30 \%$ or greater decrease in tumor size as determined by evaluation of the sum of the diameters of the target tumors, whose size was estimated using unidirectional measurement. PD was defined as $20 \%$ or greater increase in tumor size as determined by evaluation of the sum of the maximal dimensions of the target tumors. SD was defined as the absence of either PR or PD. [38] The objective response rate
(ORR) was defined as the percentage of patients who had a best response rate of CR and PR. The disease control rate (DCR) was defined as the percentage of patients who had a best response rate of CR, PR and SD. Follow-up consisted of weekly or bi-weekly blood test analyses and physical examination at each visit.

\section{Statistical analysis}

Data were expressed as the median value (range) or the mean \pm standard deviation (SD). Differences between the two groups were analyzed using the unpaired $t$ test for continuous variables, and categorical variables were analyzed using Fisher's exact test. Data were analyzed using univariate and multivariate analysis. OS was defined as the interval between the date of sorafenib and the date of death from any cause or the last follow-up date. PFS was defined as the interval between the date of sorafenib and the date of disease progression or the last follow-up date. For analysis of OS, follow-up ended at the time of death from any cause, censoring the remaining patients at the last follow-up visit. For analysis of PFS, follow-up was terminated at the time of first radiologically confirmed tumor progression; other patients were censored at their last follow-up visit and at the time of death from any cause without tumor progression. The treatment duration of sorafenib was calculated from the date of treatment commencement until treatment termination or last follow-up date, including times of interruptions. The cumulative OS and PFS rates between the two groups were calculated using the Kaplan-Meier method, and tested using the log-rank test. The Cox proportional hazard model was used for multivariate analysis of factors with $P<0.1$ in univariate analysis. Values of $P<0.05$ were considered to be statistically significant.

\section{Propensity score analysis}

To overcome biases due to the different distribution of covariates between aged group and control group, a one-to-one match was created using propensity score analysis. [39] Clinical variables entered in the propensity model were gender, C-P score, cause of liver disease, BCLC stage, ECOG PS, aspartate aminotransferase (AST), alanine aminotransferase (ALT) and initial dose of sorafenib. Subsequently, a one-to-one match between these two groups was obtained by using the nearest-neighbor matching method. [39]

\section{Results}

\section{Clinical characteristics}

The baseline clinical characteristics of the two groups (the elderly group [n=179] and the control group $[\mathrm{n}=279]$ ) are shown in table 1 . There were male 
and Child-Pugh A predominance in both groups. There was a significantly lower positivity rate for hepatitis B surface antigen, poorer PS, lower BW, lower hemoglobin level, higher serum creatinine level and lower levels of hepatobiliary enzymes such as alkaline phosphatase (ALP) and gamma glutamyl transpeptidase (GGT) in the aged group. In terms of previous therapies for HCC, TACE was most commonly performed procedure in both groups. Initial sorafenib dose of $800 \mathrm{mg} /$ day was administered in 51 patients $(28.5 \%)$ in the aged group and 132 patients $(47.3 \%)$ in the control group $(P<0.001)$, whereas initial dose of sorafenib based on BW tended to be lower in the aged group than in the control group $(P=0.057)$. Regarding BCLC stage, no significant difference in the two groups was observed $(P=0.921)$. Sixty six patients $(36.9 \%)$ in the aged group and 129 patients $(46.2 \%)$ in the control group had extrahepatic metastases with the most common sites being bone and lung.

\section{Comparison of OS and PFS rates in the two groups}

The median follow-up periods after sorafenib therapy were 7.5 months (range, 0.7-39.1 months) in the aged group and 7.6 months (range, 0.3-46.2 months) in the control group. The median OS intervals were 9.7 months (95\% confidence interval [CI], 7.5-12.0 months) in the aged group and 8.2 months (95\% CI, 6.9-9.6 months) in the control group $(P=0.641)$. (Fig. 1) The median PFS intervals were 3.8 months (95\% CI, 2.9-4.6 months) in the aged group and 3.3 months (95\% CI, 3.0-3.6 months) in the control group $(P=0.068)$. (Fig. 2$)$

Table I. Baseline characteristics between the aged group and the control group.

\begin{tabular}{|c|c|c|c|}
\hline Variables & Aged group $(n=179)$ & Control group $(n=279)$ & $P$ value \\
\hline Age (years) & $79.4 \pm 3.3$ & $64.1 \pm 7.6$ & $<0.001^{\mathrm{a}}$ \\
\hline Gender, male/female & $136 / 43$ & $233 / 46$ & $0.053^{\mathrm{b}}$ \\
\hline Body weight (kg) & $56.6 \pm 11.0$ & $60.4 \pm 12.0$ & $0.001^{\mathrm{a}}$ \\
\hline Child-Pugh A / B & $152 / 27$ & $222 / 57$ & $0.174^{\mathrm{b}}$ \\
\hline \multicolumn{4}{|l|}{ Causes of liver disease } \\
\hline $\mathrm{B} / \mathrm{C} /$ non $\mathrm{B}$ and non $\mathrm{C} / \mathrm{B}$ and $\mathrm{C}$ & $6 / 124 / 47 / 2$ & $62 / 140 / 74 / 3$ & $<0.001^{\mathrm{b}}$ \\
\hline BCLC stage $B / C$ & $63 / 116$ & $100 / 179$ & $0.921^{b}$ \\
\hline ECOG PS, $0 / 1 / 2$ & $117 / 54 / 8$ & $229 / 44 / 6$ & $<0.001^{\mathrm{b}}$ \\
\hline Portal vein invasion, yes/no & $36 / 143$ & $71 / 208$ & $0.214^{\mathrm{b}}$ \\
\hline Extrahepatic metastasis, yes/no & $66 / 113$ & $129 / 150$ & $0.053 \mathrm{~b}$ \\
\hline \multicolumn{4}{|l|}{ Previous therapies for HCC, yes/no } \\
\hline Transarterial chemoembolization & $162 / 17$ & $223 / 56$ & $0.003^{b}$ \\
\hline Ablative therapies (RFA or PEI) & $107 / 72$ & $122 / 157$ & $0.001^{b}$ \\
\hline Surgical resection & $31 / 148$ & $59 / 220$ & $0.337 \mathrm{~b}$ \\
\hline AST (IU/L) & $64.6 \pm 57.1$ & $70.3 \pm 69.1$ & $0.356^{\mathrm{a}}$ \\
\hline ALT (IU/L) & $43.9 \pm 34.1$ & $51.3 \pm 43.6$ & $0.054^{\mathrm{a}}$ \\
\hline Total bilirubin (mg/dL) & $0.92 \pm 0.44$ & $1.04 \pm 1.87$ & $0.386^{\mathrm{a}}$ \\
\hline $\operatorname{Albumin}(\mathrm{g} / \mathrm{dL})$ & $3.48 \pm 0.46$ & $3.53 \pm 0.53$ & $0.309 a$ \\
\hline $\operatorname{ALP}(\mathrm{IU} / \mathrm{L}) \mathrm{c}$ & $434.1 \pm 228.3$ & $539.3 \pm 445.9$ & $0.004^{\mathrm{a}}$ \\
\hline GGT $(\mathrm{IU} / \mathrm{L})^{\mathrm{d}}$ & $103.7 \pm 120.3$ & $171.0 \pm 194.8$ & $<0.001^{\mathrm{a}}$ \\
\hline $\mathrm{LDHe}^{\mathrm{e}}$ & $251.6 \pm 75.9$ & $254.8 \pm 105.8$ & $0.735^{\mathrm{a}}$ \\
\hline Serum creatinine $(\mathrm{mg} / \mathrm{dL})^{\mathrm{f}}$ & $0.97 \pm 0.47$ & $0.86 \pm 0.64$ & $0.036^{\mathrm{a}}$ \\
\hline Prothrombin time $(\%) \mathrm{g}$ & $85.6 \pm 19.4$ & $86.8 \pm 17.8$ & $0.506^{\mathrm{a}}$ \\
\hline Hemoglobin $(\mathrm{g} / \mathrm{dL})^{\mathrm{h}}$ & $11.7 \pm 1.9$ & $12.4 \pm 2.4$ & $0.002^{\mathrm{a}}$ \\
\hline Platelets $\left(\times 10^{4} / \mathrm{mm}^{3}\right)^{\mathrm{i}}$ & $12.8 \pm 5.7$ & $13.5 \pm 6.0$ & $0.183^{a}$ \\
\hline $\operatorname{AFP}(n g / m L) j$ & $14679 \pm 111897$ & $12459 \pm 56756$ & $0.773^{a}$ \\
\hline $\mathrm{DCP}(\mathrm{mAU} / \mathrm{mL})^{\mathrm{k}}$ & $21678 \pm 142635$ & $18709 \pm 63674$ & $0.796^{\mathrm{a}}$ \\
\hline \multicolumn{4}{|l|}{ Initial dose of sorafenib (mg/day) } \\
\hline $800 \mathrm{mg} / 600 \mathrm{mg} / 400 \mathrm{mg} / 200 \mathrm{mg}$ & $51 / 0 / 120 / 8$ & $132 / 2 / 134 / 11$ & $<0.001^{\mathrm{b}}$ \\
\hline Initial dose of sorafenib based on BW $(\mathrm{mg} / \mathrm{kg})$ & $9.2 \pm 3.9$ & $9.9 \pm 3.9$ & $0.057 \mathrm{a}$ \\
\hline
\end{tabular}

Data are expressed as number or mean \pm standard deviation. BCLC; Barcelona Clinic Liver Cancer, ECOG PS; Eastern Cooperative Oncology Group Performance Status, HCC; hepatocellular carcinoma, RFA; radiofrequency ablation, PEI; percutaneous ethanol injection, AST; aspartate aminotransferase, ALT; alanine aminotransferase, ALP; alkaline phosphatase, GGT; gamma glutamyl transpeptidase, LDH; lactose dehyrogenase, AFP; alpha-fetoprotein, DCP; des- $\gamma$-carboxy prothrombin, BW; body weight, a; unpaired $\mathrm{t}$ test, $\mathrm{b}$; Fisher's exact test, c; missing values, $\mathrm{n}=9$, d; missing values, $\mathrm{n}=8$, e; missing values, $\mathrm{n}=19$, f; missing values, $\mathrm{n}=1$, $\mathrm{g}$; missing values, $\mathrm{n}=5$, $\mathrm{h}$; missing values, $\mathrm{n}=1$, i; missing values, $n=1$, j; missing values, $n=9$, $k$; missing values, $n=17$ 


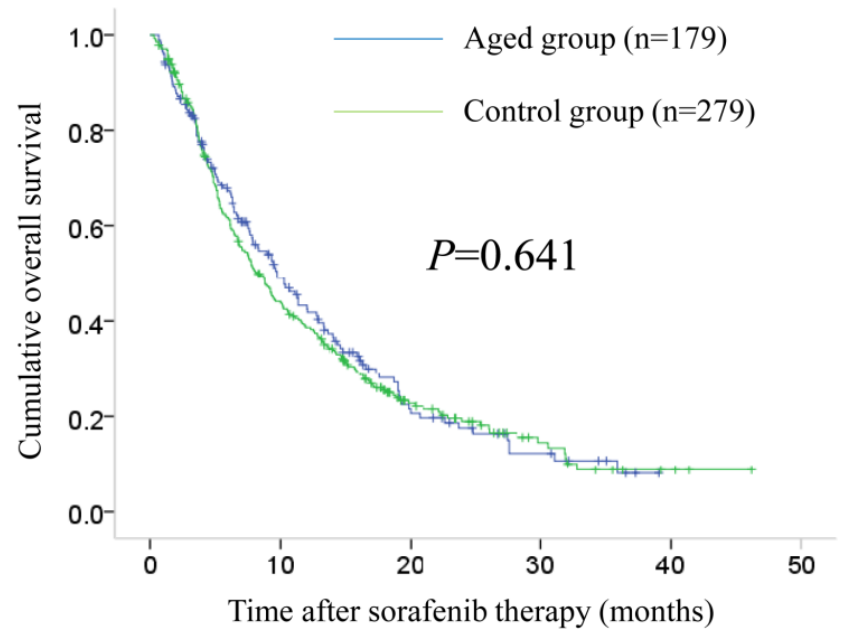

Figure I. Cumulative overall survival (OS) in the aged group $(n=179)$ and the control group $(n=279)$. The median OS intervals were 9.7 months (95\% confidence interval [Cl], 7.5-12.0 months) in the aged group and 8.2 months $(95 \% \mathrm{Cl}, 6.9-9.6$ months) in the control group $(P=0.64 \mathrm{I})$.

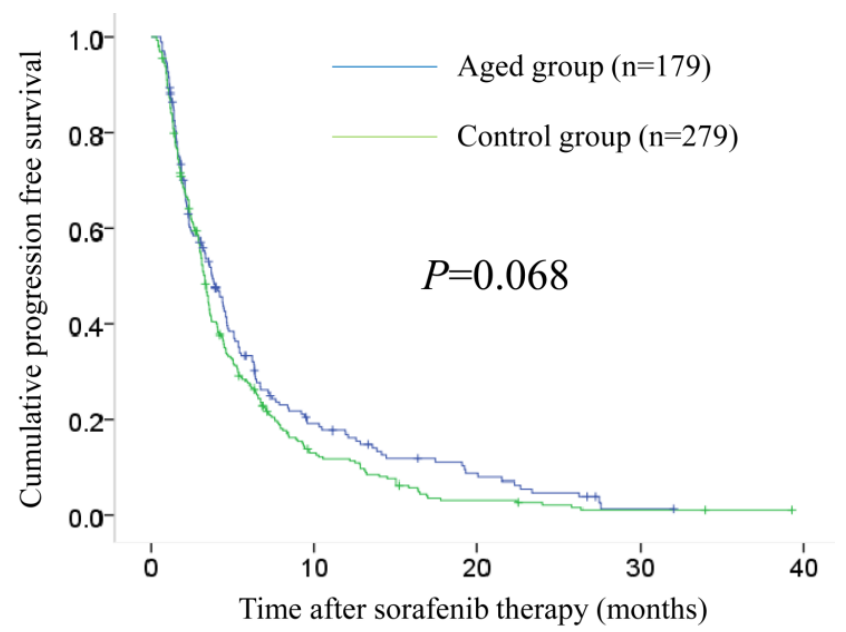

Figure 2. Cumulative progression free survival (PFS) in the aged group $(n=179)$ and the control group $(n=279)$. The median PFS intervals were 3.8 months ( $95 \% \mathrm{Cl}$, 2.9-4.6 months) in the aged group and 3.3 months $(95 \%$ $\mathrm{Cl}, 3.0-3.6$ months) in the control group $(P=0.068)$.

Treatment duration, treatment discontinuation rate and dose reduction rate in the two groups

In patients with initial dose of sorafenib of 800 $m g /$ day $(n=51$ in the aged group and $n=132$ in the control group), the median treatment durations were 3.1 months (range, 0.1-30.0 months) in the aged group and 3.2 months (range, 0.2-40.4 months) in the control group $(P=0.629)$. Treatment discontinuation rates were $90.2 \%(46 / 51)$ in the aged group and $92.4 \%$ $(122 / 132)$ in the control group $(P=0.764)$. Dose reduction rates were $62.7 \%(32 / 51)$ in the aged group and $57.6 \%(76 / 132)$ in the control group $(P=0.616)$.

In patients with reduced initial dose of sorafenib $(n=128$ in the aged group and $n=147$ in the control group), the median treatment durations were 3.3 months (range, 0.1-32.1 months) in the aged group and 3.8 months (range, 0.1-29.0 months) in the control group $(P=0.381)$. Treatment discontinuation rates were $89.8 \%(115 / 128)$ in the aged group and $89.1 \%$ $(131 / 147)$ in the control group $(P>0.999)$. Dose reduction rates were $42.2 \%(54 / 128)$ in the aged group and $29.9 \%(44 / 147)$ in the control group $(P=0.043)$, suggesting that aged group patients with reduced initial dose of sorafenib had significantly higher dose reduction rate than control group patients.

\section{Treatment tumor response rate}

The best treatment tumor response rates during follow-up period were: CR in 4 patients, $P R$ in 23, SD in 61, PD in 50 and not evaluated (NE) in 41, respectively, in the aged group; CR in 2 patients, PR in 38, SD in 97, PD in 98 and NE in 44, respectively, in the control group. The objective response rates (ORRs) were $15.1 \%$ (27 out of 179 patients) in the aged group and $14.3 \%$ (40 out of 279 patients) in the control group $(P=0.892)$. The disease control rates (DCRs) were $49.2 \%$ (88 out of 179 patients) in the aged group and $49.1 \%$ (137 out of 279 patients) in the control group $(P>0.999)$. (Table 2)

Table 2. Best treatment response rate in the aged group and the control group.

\begin{tabular}{llll}
\hline & Aged group & Control group & $P$ valuea \\
\hline Complete response & $4(2.2 \%)$ & $2(0.7 \%)$ & \\
Partial response & $23(12.8 \%)$ & $38(13.6 \%)$ & \\
Stable disease & $61(34.1 \%)$ & $97(34.8 \%)$ & \\
Progressive disease & $50(27.9 \%)$ & $98(35.1 \%)$ & \\
Unavailable response & $41(22.9 \%)$ & $44(15.8 \%)$ & \\
Disease control rate & $88 / 179(49.2 \%)$ & $137 / 279(49.1 \%)$ & $>0.999$ \\
Objective response rate & $27 / 179(15.1 \%)$ & $40 / 279(14.3 \%)$ & 0.892 \\
\hline a. Fisher's exact test & & &
\end{tabular}

a; Fisher's exact test

\section{Treatment response according to Edmondson grade}

In HCC patients with Edmondson grade I $(n=29$; $\mathrm{n}=16$ in the aged group and $\mathrm{n}=13$ in the control group), the ORRs were $18.8 \%(3 / 16)$ in the aged group and $30.8 \%(4 / 13)$ in the control group $(P=0.667)$, while the DCRs were $62.5 \%(10 / 16)$ in the aged group and $76.9 \%(10 / 13)$ in the control group $(P=0.454)$. In HCC patients with Edmondson grade II $(n=37 ; n=13$ in the aged group and $n=24$ in the control group), the ORRs were $23.1 \%(3 / 13)$ in the aged group and $4.2 \%(1 / 24)$ in the control group $(P=0.115)$, while the DCRs were $46.2 \%(6 / 13)$ in the aged group and $29.2 \%(7 / 24)$ in the control group $(P=0.472)$. In HCC patients with Edmondson grade III $(n=23 ; n=4$ in the aged group and $n=19$ in the control group), the ORRs were $25.0 \%$ $(1 / 4)$ in the aged group and $5.3 \%(1 / 19)$ in the control group $(P=0.324)$, while the DCRs were $25.0 \%(1 / 4)$ in the aged group and $36.8 \%(7 / 19)$ in the control group $(P>0.999)$. 


\section{Univariate and multivariate analyses of factors contributing to OS}

In the univariate analysis, Child-Pugh classification $(P<0.001)$, BCLC stage $(P<0.001)$, portal vein invasion $(P<0.001)$, extrahepatic spread $(P<0.001)$, EOCG PS $(P=0.001)$, AST $\geq 50 \mathrm{IU} / \mathrm{L}(P<0.001)$, ALP $\geq 400 \mathrm{IU} / \mathrm{L}(P<0.001)$, GGT $\geq 90 \mathrm{IU} / \mathrm{L}(P<0.001)$, lactose dehydrogenase $(\mathrm{LDH}) \geq 240 \quad \mathrm{IU} / \mathrm{L} \quad(P<0.001)$, alpha-fetoprotein $(\mathrm{AFP}) \geq 200 \mathrm{ng} / \mathrm{mL}(P<0.001)$ and des- $\gamma$-carboxy prothrombin $(\mathrm{DCP}) \geq 700 \mathrm{mAU} / \mathrm{mL}$ $(P<0.001)$ were significant factors contributing to OS. (Table 3) In the multivariate analysis involving 12 factors with $P<0.1$ in the univariate analyses, Child-Pugh classification $(P=0.005)$, causes of liver disease (viral) $(P=0.001)$, portal vein invasion $(P=0.007)$, extrahepatic spread $(P=0.002)$, GGT $\geq 90$ $\mathrm{IU} / \mathrm{L}(P<0.001), \mathrm{LDH} \geq 240 \mathrm{IU} / \mathrm{L}(P<0.001), \mathrm{AFP} \geq 200$ $\mathrm{ng} / \mathrm{mL}(P<0.001)$ and $\mathrm{DCP} \geq 700 \mathrm{mAU} / \mathrm{mL}(P=0.002)$ were significant factors contributing to OS. The hazard ratios (HRs) and 95\% CIs for these factors are detailed in table 4.

\section{Univariate and multivariate analyses of factors contributing to PFS}

In the univariate analysis, Child-Pugh classification $(P=0.002)$, BCLC stage $(P=0.023)$, portal vein invasion $(P=0.005)$, AST $\geq 50 \mathrm{IU} / \mathrm{L}(P=0.002), \mathrm{ALP} \geq 400$ $\mathrm{IU} / \mathrm{L}(P=0.001), \mathrm{GGT} \geq 90 \mathrm{IU} / \mathrm{L}(P<0.001), \mathrm{LDH} \geq 240$ $\mathrm{IU} / \mathrm{L}(P<0.001), \mathrm{AFP} \geq 200 \mathrm{ng} / \mathrm{mL}(P<0.001)$ and $\mathrm{DCP}$ $\geq 700 \mathrm{mAU} / \mathrm{mL}(P<0.001)$ were significant factors associated with PFS. (Table 3 ) In the multivariate analysis involving 10 factors with $P<0.1$ the univariate analysis, Child-Pugh classification $(P=0.031)$, GGT $\geq 90 \mathrm{IU} / \mathrm{L}(P=0.008), \mathrm{LDH} \geq 240 \mathrm{IU} / \mathrm{L}(P=0.043)$, AFP $\geq 200 \mathrm{ng} / \mathrm{mL}(P=0.009)$ and $\mathrm{DCP} \geq 700 \mathrm{mAU} / \mathrm{mL}$ $(P=0.009)$ were significant factors linked to PFS. The HRs and $95 \%$ CIs for these factors are detailed in table 4 .

\section{Causes of death in the two groups}

One hundred and twenty seven patients $(70.9 \%)$ in the aged group and $215(77.1 \%)$ patients in the control group died during the follow-up period. The causes of death in the aged group were as follows: HCC progression (90 patients); liver failure (19 patients); miscellaneous (15 patients); and unknown causes ( 3 patients). In the control group the causes of death were: HCC progression (178 patients); liver failure (13 patients); miscellaneous (17 patients); and unknown causes (7 patients).

Table 3. Univariate analyses of factors contributing to overall survival (OS) and progression free survival (PFS).

\begin{tabular}{|c|c|c|c|}
\hline & & OS & PFS \\
\hline Variables & $\mathrm{n}$ & $P$ valuea & $P$ valuea \\
\hline Age ( $\geq 75$ years), yes $/$ no & $179 / 279$ & 0.641 & 0.068 \\
\hline Gender (male), yes/no & $369 / 89$ & 0.353 & 0.828 \\
\hline Child-Pugh classification, A/B & $374 / 84$ & $<0.001$ & 0.002 \\
\hline BCLC stage, B/C & $163 / 295$ & $<0.001$ & 0.023 \\
\hline Causes of liver disease (viral), yes/no & $337 / 121$ & 0.054 & 0.134 \\
\hline Portal vein invasion, yes/no & $107 / 351$ & $<0.001$ & 0.005 \\
\hline Extrahepatic spread, yes/no & $195 / 263$ & $<0.001$ & 0.394 \\
\hline EOCG PS 0, yes/no & $346 / 112$ & 0.001 & 0.291 \\
\hline AST $(\geq 50 \mathrm{IU} / \mathrm{L})$, yes $/$ no & $251 / 207$ & $<0.001$ & 0.002 \\
\hline $\operatorname{ALT}(\geq 50 \mathrm{IU} / \mathrm{L})$, yes/no & $206 / 252$ & 0.270 & 0.346 \\
\hline $\operatorname{ALP}(\geq 400 \mathrm{IU} / \mathrm{L})$, yes $/ \mathrm{no}^{\mathrm{b}}$ & $220 / 229$ & $<0.001$ & 0.001 \\
\hline GGT ( $\geq 90 \mathrm{IU} / \mathrm{L})$, yes/noc & $209 / 241$ & $<0.001$ & $<0.001$ \\
\hline $\mathrm{LDH}(\geq 240 \mathrm{IU} / \mathrm{L})$, yes $/$ nod $^{\mathrm{d}}$ & $202 / 237$ & $<0.001$ & $<0.001$ \\
\hline Platelets $\left(\geq 12 \times 10^{4} / \mathrm{mm}^{3}\right)$, yes $/$ noe & $224 / 233$ & 0.259 & 0.658 \\
\hline $\operatorname{AFP}(\geq 200 \mathrm{ng} / \mathrm{mL})$, yes $/$ nof $^{f}$ & $211 / 238$ & $<0.001$ & $<0.001$ \\
\hline $\mathrm{DCP}(\geq 700 \mathrm{mAU} / \mathrm{mL})$, yes $/$ nog & $217 / 224$ & $<0.001$ & $<0.001$ \\
\hline $\begin{array}{l}\text { Initial dose of sorafenib }(800 \mathrm{mg} / \text { day }) \\
\text { yes/no }\end{array}$ & $183 / 275$ & 0.950 & 0.788 \\
\hline Initial dose of sorafenib based on $B W \geq 8.4$ & $222 / 236$ & 0.470 & 0.187 \\
\hline
\end{tabular}
$\mathrm{mg} / \mathrm{kg} /$ day, yes $/$ no

BCLC; Barcelona Clinic Liver Cancer, ECOG PS; Eastern Cooperative Oncology Group Performance Status, AST; aspartate aminotransferase, ALT; alanine aminotransferase, ALP; alkaline phosphatase, GGT; gamma glutamyl transpeptidase, $\mathrm{LDH}$; lactose dehyrogenase, AFP; alpha-fetoprotein, DCP; des- $\gamma$-carboxy prothrombin, $\mathrm{BW}$; body weight, a, log-rank test, b; missing values, $\mathrm{n}=9$, c; missing values, $\mathrm{n}=8$, d; missing values, $\mathrm{n}=19$,, ; missing values, $\mathrm{n}=1, \mathrm{f}$; missing values, $\mathrm{n}=9$, $\mathrm{g}$; missing values, $\mathrm{n}=17$

Table 4. Multivariate analyses of factors contributing to overall survival (OS) and progression free survival (PFS).

\begin{tabular}{|c|c|c|c|c|c|c|}
\hline & OS & & & PFS & & \\
\hline Variables & HR & $95 \% \mathrm{CI}$ & $P$ value & HR & $95 \% \mathrm{CI}$ & $P$ value \\
\hline Age ( $\geq 75$ years $)$ & & & & 0.926 & $0.746-1.151$ & 0.490 \\
\hline Child-Pugh classification, A/B & 0.658 & $0.491-0.882$ & 0.005 & 0.741 & $0.564-0.972$ & 0.031 \\
\hline BCLC stage, B/C & 0.952 & $0.632-1.434$ & 0.815 & 0.840 & $0.660-1.070$ & 0.158 \\
\hline Causes of liver disease (viral) & 0.628 & $0.472-0.836$ & 0.001 & & & \\
\hline Portal vein invasion & 0.657 & $0.485-0.891$ & 0.007 & 0.947 & $0.719-1.248$ & 0.699 \\
\hline Extrahepatic spread & 0.599 & $0.433-0.828$ & 0.002 & & & \\
\hline EOCG PS, $0 / 1,2$ & 0.785 & $0.581-1.060$ & 0.115 & & & \\
\hline AST ( $\geq 50 \mathrm{IU} / \mathrm{L})$ & 1.140 & $0.858-1.514$ & 0.368 & 1.025 & $0.809-1.298$ & 0.840 \\
\hline $\operatorname{ALP}(\geq 400 \mathrm{IU} / \mathrm{L})$ & 0.960 & $0.740-1.246$ & 0.760 & 1.008 & $0.799-1.271$ & 0.946 \\
\hline GGT $(\geq 90 \mathrm{IU} / \mathrm{L})$ & 0.609 & $0.472-0.786$ & $<0.001$ & 0.729 & $0.578-0.921$ & 0.008 \\
\hline LDH ( $\geq 240 \mathrm{IU} / \mathrm{L})$ & 0.558 & $0.434-0.719$ & $<0.001$ & 0.794 & $0.635-0.992$ & 0.043 \\
\hline $\mathrm{AFP}(\geq 200 \mathrm{ng} / \mathrm{mL})$ & 0.601 & $0.474-0.763$ & $<0.001$ & 0.749 & $0.604-0.930$ & 0.009 \\
\hline $\mathrm{DCP}(\geq 700 \mathrm{mAU} / \mathrm{mL})$ & 0.676 & $0.529-0.863$ & 0.002 & 0.766 & $0.616-0.952$ & 0.016 \\
\hline
\end{tabular}

HR; hazard ratio, CI; confidence interval, BCLC; Barcelona Clinic Liver Cancer, ECOG PS; Eastern Cooperative Oncology Group Performance Status, AST; aspartate aminotransferase, ALP; alkaline phosphatase, GGT; gamma glutamyl transpeptidase, LDH; lactose dehyrogenase, AFP; alpha-fetoprotein, DCP; des- $\gamma$-carboxy prothrombin, a, Cox proportional hazard model. 


\section{Serious adverse events (SAEs)}

Grade 3 or more SAEs as defined by CTCAE were observed in 51 patients $(28.5 \%)$ in the elderly group and 69 patients $(24.7 \%)$ in the control group $(P=0.385)$ : rash $(5.7 \%[10 / 175]$ vs. $2.2 \%$ [6/274], $P=0.066)$, hand-foot syndrome $(6.9 \%[12 / 175]$ vs. $4.4 \%$ $[12 / 275], P=0.285)$, diarrhea $(2.3 \%[4 / 174]$ vs. $2.2 \%$ [6/277], $P>0.999)$, fever (1.1\% [2/177] vs. $1.4 \%$ [4/278], $P>0.999)$, fatigue $(4.0 \%[7 / 175]$ vs. $2.5 \%[7 / 276]$, $P=0.412)$, hypertension $(0.6 \%[1 / 175]$ vs. $1.8 \%$ [5/276], $P=0.412)$, gastrointestinal bleeding $(1.7 \%[3 / 175]$ vs. $1.4 \%$ [4/276], $P>0.999)$, liver damage (9.7\% [17/175] vs. $13.0 \%[36 / 276], P=0.299)$ and lung injury $(4.0 \%$ [7/174] vs. $0 \%$ [0/276], $P=0.001$ ). (Table 5)

Table 5. Treatment related serious adverse events of grade 3 or more in the aged group and the control group.

\begin{tabular}{|c|c|c|c|}
\hline \multirow[t]{2}{*}{ Adverse events } & \multirow{2}{*}{$\begin{array}{l}\text { Aged group } \\
\text { Grade } 3 \text { or more } \\
\text { SAEs }\end{array}$} & \multicolumn{2}{|l|}{ Control group } \\
\hline & & $\begin{array}{l}\text { Grade } 3 \text { or more } \\
\text { SAEs }\end{array}$ & $\begin{array}{l}P \\
\text { valuea }\end{array}$ \\
\hline Overall & $51 / 179(28.5 \%)$ & $69 / 279(24.7 \%)$ & 0.385 \\
\hline Rash $^{\mathrm{b}}$ & $10 / 175(5.7 \%)$ & $6 / 274(2.2 \%)$ & 0.066 \\
\hline Hand foot syndromec & $12 / 175(6.9 \%)$ & $12 / 275(4.4 \%)$ & 0.285 \\
\hline Diarrhea $^{d}$ & $4 / 174(2.3 \%)$ & $6 / 277(2.2 \%)$ & $>0.999$ \\
\hline Fevere & $2 / 177(1.1 \%)$ & $4 / 278(1.4 \%)$ & $>0.999$ \\
\hline Fatiguef & $7 / 175(4.0 \%)$ & $7 / 276(2.5 \%)$ & 0.412 \\
\hline Hypertensiong & $1 / 175(0.6 \%)$ & $5 / 276(1.8 \%)$ & 0.412 \\
\hline $\begin{array}{l}\text { Gastrointestinal } \\
\text { bleedingh }\end{array}$ & $3 / 175(1.7 \%)$ & $4 / 276(1.4 \%)$ & $>0.999$ \\
\hline Liver damage $\mathrm{e}^{\mathrm{i}}$ & $17 / 175(9.7 \%)$ & $36 / 276(13.0 \%)$ & 0.299 \\
\hline Lung injuryj & $7 / 174(4.0 \%)$ & $0 / 276(0 \%)$ & 0.001 \\
\hline
\end{tabular}

\section{Subgroup analyses according to Child-Pugh classification}

In patients with Child-Pugh A ( $\mathrm{n}=152$ in the aged group and $n=222$ in the control group), the median OS intervals were 11.3 months (95\% CI, 9.0-13.6 months) in the aged group and 9.3 months $(95 \% \mathrm{CI}$, 7.0-11.7 months) in the control group $(P=0.690)$. The median PFS intervals were 4.2 months (95\% CI, 3.5-5.0 months) in the aged group and 3.3 months (95\% CI, 3.0-3.6 months) in the control group ( $P=0.047)$, suggesting that the aged group patients with Child-Pugh A had significantly higher PFS rate compared with the control group. In patients with Child-Pugh $B(n=27$ in the aged group and $n=57$ in the control group), the median OS intervals were 4.9 months (95\% CI, 2.8-7.0 months) in the aged group and 4.4 months (95\% CI, 3.3-5.4 months) in the control group $(P=0.704)$. The median PFS intervals were 1.6 months ( $95 \%$ CI, 0.2-3.0 months) in the aged group and 2.6 months $(95 \% \mathrm{CI}$, $1.3-3.8$ months) in the control group $(P=0.554)$.
Subgroup analyses according to BCLC stage

In patients with BCLC stage $B(n=63$ in the aged group and $n=100$ in the control group), the median OS intervals were 14.6 months (95\% CI, 9.6-19.7 months) in the aged group and 15.0 months (95\% CI, 11.9-18.0 months) in the control group $(P=0.530)$. The median PFS intervals were 3.8 months (95\% CI, 2.6-5.1 months) in the aged group and 4.2 months (95\% CI, 3.2-5.3 months) in the control group ( $P=0.768)$. In patients with BCLC stage $C(n=116$ in the aged group and $n=179$ in the control group), the median OS intervals were 7.9 months (95\% CI, 5.4-10.3 months) in the aged group and 6.1 months $(95 \% \mathrm{CI}, 5.0-7.2$ months) in the control group ( $P=0.269)$. The median PFS intervals were 3.6 months $(95 \% \mathrm{CI}, 2.4-4.7$ months) in the aged group and 2.9 months $(95 \% \mathrm{CI}$, 2.4-3.3 months) in the control group $(P=0.046)$, indicating that the aged group patients with BCLC stage $C$ had significantly higher PFS rate than the control group patients.

\section{Subgroup analyses according to initial dose of sorafenib}

We further analysed clinical outcomes according to initial dose of sorafenib since the proportion of patients with initial dose of sorafenib of $800 \mathrm{mg}$ /day in the aged group was significantly lower than that in the control group. In patients with initial dose of sorafenib of $800 \mathrm{mg} /$ day $(\mathrm{n}=51$ in the aged group and $\mathrm{n}=132$ in the control group), the median OS intervals were 12.0 months (95\% CI, 7.8-16.3 months) in the aged group and 7.1 months (95\% CI, 5.3-8.9 months) in the control group $(P=0.332)$. The median PFS intervals were 4.2 months (95\% CI, 3.3-5.1 months) in the aged group and 3.2 months $(95 \% \mathrm{CI}, 2.9-3.5$ months) in the control group $(P=0.079)$. In patients with reduced initial dose of sorafenib $(n=128$ in the aged group and $n=147$ in the control group), the median OS intervals were 9.3 months (95\% CI, 7.1-11.6 months) in the aged group and 9.2 months $(95 \% \mathrm{CI}$, 6.9-11.6 months) in the control group $(P=0.850)$. The median PFS intervals were 3.6 months (95\% CI, 2.7-4.5 months) in the aged group and 3.4 months (95\% CI, 2.9-3.9 months) in the control group $(P=0.253)$.

\section{Baseline characteristics and clinical outcomes in the aged and control groups after propensity score matching}

Baseline characteristics in the two groups (aged group: $n=132$, control group: $n=132$ ) after propensity score matching are demonstrated in Table 6. In all analysed variables, no significant differences were observed. The median OS intervals were 10.7 months (95\% CI, 8.0-13.4 months) in the aged group and 9.5 months (95\% CI, 6.6-12.4 months) in the control group 
( $P=0.898$ ). (Fig. 3) The median PFS intervals were 3.8 months (95\% CI, 2.6-5.1 months) in the aged group and 3.8 months (95\% CI, 2.9-4.8 months) in the control group $(P=0.407)$. (Fig. 4 )

\section{Clinical outcome in the two groups according to different cut-off age}

When using cut-off age of 80 years $(n=81$ in patients aged $\geq 80$ years and $n=377$ in patients aged $<80$ years), the median OS intervals were 9.3 months (95\% CI, 7.4-11.3 months) in the aged group and 8.8 months (95\% CI, 7.5-10.1 months) in the control group $(P=0.827)$, while the median PFS intervals were 3.8

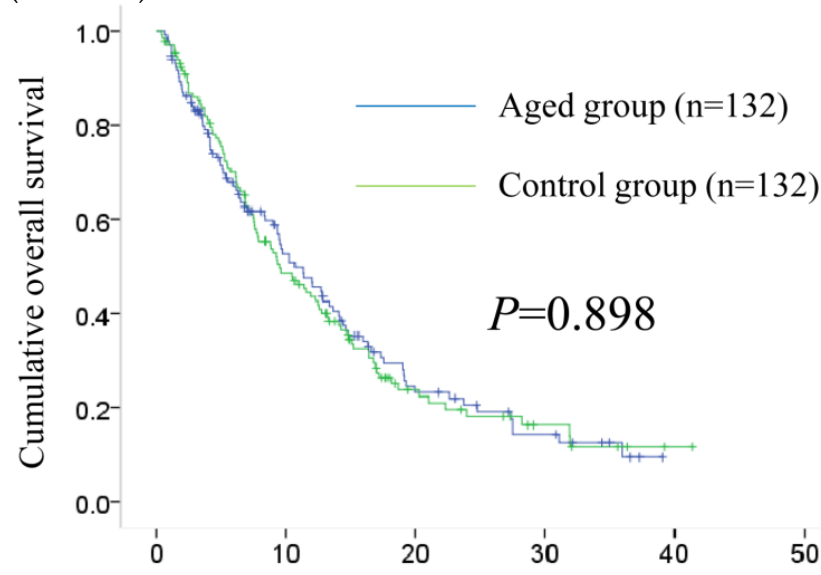

Time after sorafenib therapy (months)

Figure 3. Cumulative overall survival (OS) in the aged group $(n=132)$ and the control group $(n=132)$ after propensity score matching. The median OS intervals were 10.7 months $(95 \% \mathrm{Cl}, 8.0-13.4$ months) in the aged group and 9.5 months $(95 \% \mathrm{Cl}, 6.6-12.4$ months $)$ in the control group $(P=0.898)$. months (95\% CI, 2.2-5.4 months) in the aged group and 3.4 months (95\% CI, 3.1-3.7 months) in the control group $(P=0.668)$. When using cut-off age of 70 years $(n=249$ in patients aged $\geq 70$ years and $n=209$ in patients aged $<70$ years), the median OS intervals were 10.1 months (95\% CI, 8.5-11.8 months) in the aged group and 7.7 months (95\% CI, 6.2-9.2 months) in the control group $(P=0.950)$, whereas the median PFS intervals were 3.7 months (95\% CI, 3.1-4.4 months) in the aged group and 3.1 months $(95 \% \mathrm{CI}, 2.8-3.4$ months) in the control group $(P=0.046)$.

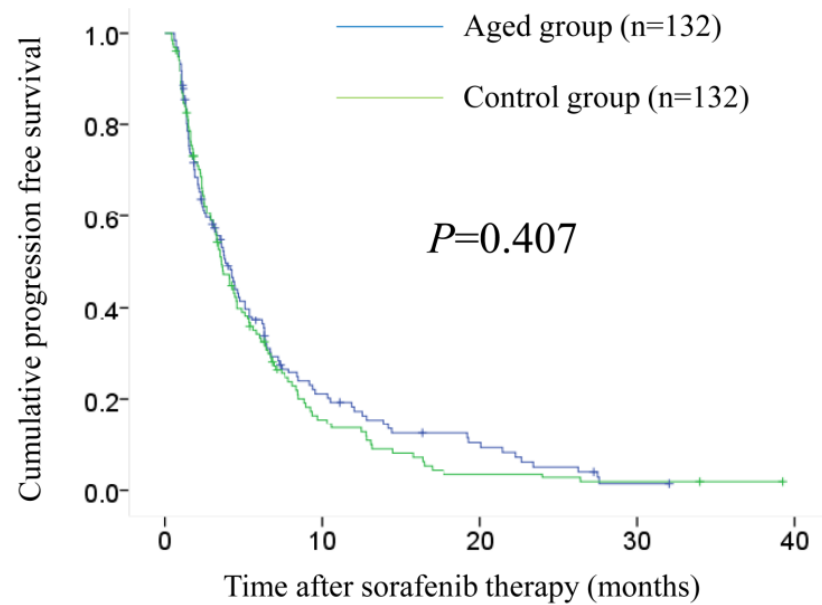

Figure 4. Cumulative progression free survival (PFS) in the aged group $(n=132)$ and the control group $(n=132)$ after propensity score matching. The median PFS intervals were 3.8 months ( $95 \% \mathrm{Cl}$, 2.9-4.8 months) in the aged group and 3.6 months ( $95 \% \mathrm{Cl}, 2.9-4.3$ months) in the control group $(P=0.407)$.

Table 6. Baseline characteristics between the aged group and the control group after propensity score matching.

\begin{tabular}{|c|c|c|c|}
\hline Variables & Aged group $(n=132)$ & Control group $(n=132)$ & $P$ value \\
\hline Age (years) & $79.4 \pm 3.3$ & $64.1 \pm 6.2$ & $<0.001^{a}$ \\
\hline Gender, male/female & $101 / 31$ & $108 / 24$ & $0.363^{b}$ \\
\hline Child-Pugh A / B & $110 / 22$ & 115 / 17 & $0.488^{b}$ \\
\hline \multicolumn{4}{|l|}{ Causes of liver disease } \\
\hline $\mathrm{B} / \mathrm{C} /$ non $\mathrm{B}$ and non $\mathrm{C} / \mathrm{B}$ and $\mathrm{C}$ & $6 / 85 / 41 / 0$ & $7 / 85 / 40$ / 0 & $>0.999 \mathrm{~b}$ \\
\hline BCLC stage $\mathrm{B} / \mathrm{C}$ & $48 / 84$ & $42 / 90$ & $0.516^{\mathrm{b}}$ \\
\hline ECOG PS, $0 / 1 / 2$ & $94 / 35 / 3$ & $95 / 34 / 3$ & $>0.999 \mathrm{~b}$ \\
\hline AST (IU/L) & $65.7 \pm 64.6$ & $60.8 \pm 35.6$ & $0.296^{a}$ \\
\hline ALT (IU/L) & $45.8 \pm 38.1$ & $48.0 \pm 35.2$ & $0.622^{a}$ \\
\hline Total bilirubin $(\mathrm{mg} / \mathrm{dL})$ & $0.93 \pm 0.45$ & $0.89 \pm 0.47$ & $0.422^{\mathrm{a}}$ \\
\hline Albumin $(\mathrm{g} / \mathrm{dL})$ & $3.50 \pm 0.46$ & $3.54 \pm 0.50$ & $0.458^{a}$ \\
\hline $\operatorname{ALP}(\mathrm{IU} / \mathrm{L}) \mathrm{c}$ & $443.9 \pm 238.0$ & $469.3 \pm 360.6$ & $0.505^{\mathrm{a}}$ \\
\hline GGT (IU/L)d & $112.6 \pm 129.0$ & $143.0 \pm 164.6$ & $0.101^{\mathrm{a}}$ \\
\hline $\mathrm{LDH}^{\mathrm{e}}$ & $248.0 \pm 76.1$ & $252.4 \pm 101.5$ & $0.696^{\mathrm{a}}$ \\
\hline Prothrombin time (\%) & $85.7 \pm 18.2$ & $88.1 \pm 16.7$ & $0.261^{\mathrm{a}}$ \\
\hline Platelets $\left(\times 10^{4} / \mathrm{mm}^{3}\right)^{\mathrm{f}}$ & $13.1 \pm 6.0$ & $13.9 \pm 6.3$ & $0.285^{a}$ \\
\hline $\operatorname{AFP}(n g / m L) g$ & $6779 \pm 26576$ & $15102 \pm 67697$ & $0.191^{\mathrm{a}}$ \\
\hline $\mathrm{DCP}(\mathrm{mAU} / \mathrm{mL})^{\mathrm{h}}$ & $13873 \pm 75599$ & $21164 \pm 80945$ & $0.457 \mathrm{a}$ \\
\hline \multicolumn{4}{|l|}{ Initial dose of sorafenib (mg/day) } \\
\hline $800 \mathrm{mg} / 600 \mathrm{mg} / 400 \mathrm{mg} / 200 \mathrm{mg}$ & $44 / 0 / 82 / 6$ & $48 / 2 / 76$ / 6 & $0.593^{b}$ \\
\hline
\end{tabular}

Data are expressed as number or mean \pm standard deviation. BCLC; Barcelona Clinic Liver Cancer, ECOG PS; Eastern Cooperative Oncology Group Performance Status, AST; aspartate aminotransferase, ALT; alanine aminotransferase, ALP; alkaline phosphatase, GGT; gamma glutamyl transpeptidase, LDH; lactose dehyrogenase, AFP; alpha-fetoprotein, DCP; des- $\gamma$-carboxy prothrombin, a; unpaired $t$ test, $b_{;}$Fisher's exact test, ${ }^{\text {; }}$ missing values, $n=7$, d; missing values, $n=6$, e; missing values, $n=11$, f; missing values, $n=1$, g; missing values, $n=2$, h; missing values, $n=8$ 


\section{Discussion}

To the best of our knowledge, this is the largest study comparing clinical outcomes and safety between aged and non-aged HCC patients treated with sorafenib. [29-32] Current guidelines for the management of HCC do not satisfy strategies according to age. $[2,3]$ Few studies assessed the clinical outcomes in HCC patients treated with sorafenib based on age. $[29,30,32]$ With the aging population, HCC in the elderly represents a significant health burden. In Japan, the proportion of elderly patients with HCC and their average age is increasing. These trends have led to a rising demand in our country for investigations related to the outcome of sorafenib therapy in elderly HCC patients: hence the reasons for the current comparative study.

In our results, the aged group patients had comparable OS rate, PFS rate, DCR and ORR as compared with the control group patients. The difference in the two groups in terms of sorafenib related SAEs of grade 3 or more did not reach significance except for the development of lung injury. In subgroup analyses, in patients with Child-Pugh A and in those with BCLC-C, the median PFS intervals in the aged group were significantly longer than those in the control group and in all other subgroup analyses, no significant difference in the two groups was observed in terms of OS and PFS. Furthermore, in the propensity score matched cohorts, no significant difference in the two groups was found in terms of OS and PFS and when using different cut-off age (80 years or 70 years), and similar results were obtained. Systemic anticancer therapy in aged patients with malignancies tends to be viewed with skepticism owing to the greater frequency of treatment related SAEs in aged than in younger patients. However, our results suggest that aged HCC patients treated with sorafenib had comparable prognosis and well tolerable drug related toxicity compared with younger HCC patients treated with sorafenib, which are in line with results reported by Jo, et al. [40] Since our study regarding effect of sorafenib on clinical outcome stratified by age is the largest that has been published so far and includes unselected cases by fourteen centers scattered throughout in Japan, our study results faithfully reflect what actually occurs in clinical practice.

The prevalence of aged subjects in our population was higher than in other previous reports. [11-26] This was possibly due to a lower proportion of patients with HBV infection who often develop HCC in younger age as compared with those with $\mathrm{HCV}$ infection and the shift towards older ages of HCC occurrence in Japan. The proportion of male patients in the aged group was almost significantly lower than that in the control group $(P=0.053)$. This may have been associated with a larger female elderly population because of their longer life expectancy. Furthermore, the observations of significantly lower hemoglobin level, lower BW and higher serum creatinine levels in the aged group of this study may well reflect the actual situations in aged HCC patients in clinical practice.

In aged group, the difference in patients with initial dose of sorafenib of $800 \mathrm{mg} /$ day and those with reduced dose of sorafenib did not reach significance in terms of OS $(P=0.445)$ and PFS $(P=0.691)$. Iavarone $\mathrm{M}$, et al reported that the effectiveness of half-dosed sorafenib may have implications for tailored therapy in HCC patients. [41] Since in aged HCC patients, high frequency of sorafenib related SAEs were expected when given an initial dose of sorafenib of 800 $\mathrm{mg} /$ day, leading to treatment discontinuation or interruptions, reduced initial dose of sorafenib can be considered in elderly patients for avoiding SAEs although further examination is needed to confirm these results.

As described earlier, in patients with Child-Pugh $A$ and in those with BCLC-C, PFS intervals in the aged group were significantly longer than that in the control group. These findings might be associated with the slower cancer growth in aged patients or to a higher susceptibility of vasculature to antiangiogenic agents in aged patients. [42] On the other hand, it is of interest that GGT level was the significant predictor linked to both OS and PFS in our multivariate analysis. Several studies reported that a high level of GGT was related to a higher incidence of HCC progression, which are in line with our results. [43, 44] As for other significant predictors observed in our multivariate analyses, our study results were consistent with previous reports. $[7,9,29,30,32,38]$

It is noteworthy that sorafenib related lung injury of grade 3 or more occurred in 7 aged patients, whereas no such lung injury was observed in the control group and 2 out of 7 died due to respiratory failure. The reasons for these results are unclear, however, during sorafenib therapy, caution should be exercised for lung injury especially in aged patients.

Although several studies have examined the predictive factors linked to the response to sorafenib in advanced HCC patients, the factors predicting a favorable response remained unclear. [45] However, recent studies demonstrated that polymorphisms of VEGF and its receptor genes may regulate angiogenesis and tumor growth and they may influence OS and PFS in HCC patients undergoing sorafeinb therapy. $[46,47]$ In addition, Lee, et al. reported that differences in the incidence of sorafenib-related hand foot skin reaction in HCC patients treated with sorafenib 
may have been caused by ethnic differences in genetic polymorphisms of the tumor necrosis factor-alpha, VEGF, and uridine diphosphate glucuronosyltransferase 1 family-polypeptide A9 genes. [48] Although such polymorphisms were not tested in the current analyses, these may be associated with clinical outcome in elderly HCC patients treated with sorafenib and in this regard, further investigations will be required.

This study included several limitations. First, our study is a retrospective observational study, although the major strength of our study is a large sample size. Second, the initial sorafenib dose varied among individual patients, leading to bias. Third, various therapies were performed after discontinuation of sorafenib in some patients, also potentially leading to bias in concerning their OS. Lastly, our study cohort is limited to Japanese patients with relatively low BW in contrast to patients in Western countries. Hence, our results cannot be extended to patients with other racial cohorts and caution should be exercised when interpreting these results. Thus, further prospective studies will be necessary. However, our results indicated that in HCC patients treated with sorafenib, life expectancy, disease progression, treatment efficacy and SAEs are unaffected by age over 75 years. In conclusion, aged HCC patients treated with sorafenib had comparable clinical outcomes compared with younger HCC patients treated with sorafenib. Sorafenib therapy for HCC should not be determined solely based on age.

\section{Acknowledgement}

The authors would like to thank all the staff in the Japanese Red Cross Liver Study group for their valuable support.

\section{Conflicts of interest} interest.

The authors declare that they have no conflicts of

\section{References}

[1] Kudo M: Radiofrequency ablation for hepatocellular carcinoma: updated review in 2010. Oncology 2010; 78: 113-124.

[2] Livraghi T, Mäkisalo H, Line PD: Treatment options in hepatocellular carcinoma today. Scand J Surg 2011; 100: 22-29.

[3] de Lope CR, Tremosini S, Forner A, et al. Management of HCC. J Hepatol 2012; 56 Suppl 1: S75-S87.

[4] El-Serag HB: Epidemiology of viral hepatitis and hepatocellular carcinoma. Gastroenterology 2012; 142: 1264-1273.

[5] Hankey BF, Ries LA, Kosary CL, et al: Partitioning linear trends in age-adjusted rates. Cancer Causes Control 2000; 11: 31-35.

[6] Cho SJ, Yoon JH, Hwang SS, Lee HS: Do young hepatocellular carcinoma patients with relatively good liver function have poorer outcomes than elderly patients? J Gastroenterol Hepatol 2007; 22: 1226-1231.

[7] Llovet JM, Ricci S, Mazzaferro V, et al. SHARP Investigators Study Group. Sorafenib in advanced hepatocellular carcinoma. N Engl J Med 2008; 359: 378-390.

[8] Abou-Alfa GK, Schwartz L, Ricci S, et al. Phase II study of sorafenib in patients with advanced hepatocellular carcinoma. J Clin Oncol 2006; 24: 4293-4300.
[9] Cheng AL, Kang YK, Chen Z, et al. Efficacy and safety of sorafenib in patients in the Asia-Pacific region with advanced hepatocellular carcinoma: a phase III randomised, double-blind, placebo-controlled trial. Lancet Oncol 2009; 10: 25-34.

[10] Fentiman IS, Tirelli U, Monfardini S, et al. Cancer in the elderly: why so badly treated? Lancet 1990; 335(8696): 1020-2.

[11] Takahashi H, Mizuta T, Kawazoe S, et al: Efficacy and safety of radiofrequency ablation for elderly hepatocellular carcinoma patients. Hepatol Res 2010; 40: 997-1005.

[12] Bove A, Bongarzoni G, Di Renzo RM, Marsili L, Chiarini S, Corbellini L: Efficacy and safety of ablative techniques in elderly HCC patients. Ann Ital Chir 2011; 82: 457-463.

[13] Massarweh NN, Park JO, Yeung RS, Flum DR: Comparative assessment of the safety and effectiveness of radiofrequency ablation among elderly medicare beneficiaries with hepatocellular carcinoma. Ann Surg Oncol 2012; 19: 1058-1065.

[14] Hiraoka A, Michitaka K, Horiike N, et al: Radiofrequency ablation therapy for hepatocellular carcinoma in elderly patients. J Gastroenterol Hepatol 2010; 25: 403-407.

[15] Kondo K, Chijiiwa K, Funagayama M, Kai M, Otani K, Ohuchida J: Hepatic resection is justified for elderly patients with hepatocellular carcinoma. World J Surg 2008; 32: 2223-2229.

[16] Oishi K, Itamoto T, Kobayashi T, et al: Hepatectomy for hepatocellular carcinoma in elderly patients aged 75 years or more. J Gastrointest Surg 2009; 13: 695-701.

[17] Huang J, Li BK, Chen GH, et al: Long-term outcomes and prognostic factors of elderly patients with hepatocellular carcinoma undergoing hepatectomy. $J$ Gastrointest Surg 2009; 13: 1627-1635.

[18] Yau T, Yao TJ, Chan P, et al: The outcomes of elderly patients with hepatocellular carcinoma treated with transarterial chemoembolization. Cancer 2009; 115: 5507-5515.

[19] Mirici-Cappa F, Gramenzi A, Santi V, et al: Treatments for hepatocellular carcinoma in elderly patients are as effective as in younger patients: a 20-year multicentre experience. Gut 2010; 59: 387-396.

[20] Yanaga K, Kanematsu T, Takenaka K, Matsumata T, Yoshida Y, Sugimachi K: Hepatic resection for hepatocellular carcinoma in elderly patients. Am J Surg 1988; 155: 238-241.

[21] Fortner JG, Lincer RM: Hepatic resection in the elderly. Ann Surg 1990; 211: 141-145

[22] Yamamoto K, Takenaka K, Matsumata T, et al: Right hepatic lobectomy in elderly patients with hepatocellular carcinoma. Hepatogastroenterology 1997; 44: 514-518.

[23] Ezaki T, Yukaya H, Ogawa Y: Evaluation of hepatic resection for hepatocellular carcinoma in the elderly. Br I Surg 1987; 74: 471-473.

[24] Nagasue N, Chang YC, Takemoto $Y$, Taniura H, Kohno H, Nakamura T: Liver resection in the aged (seventy years or older) with hepatocellular carcinoma. Surgery 1993; 113: 148-154.

[25] Takenaka K, Shimada M, Higashi H, et al: Liver resection for hepatocellular carcinoma in the elderly. Arch Surg 1994; 129: 846-850.

[26] Miyagawa S, Makuuchi M, Kawasaki S, Kakazu T: Criteria for safe hepatic resection. Am J Surg 1995; 169: 589-594.

[27] Nishikawa H, Osaki Y, Iguchi E, et al. Percutaneous radiofrequency ablation for hepatocellular carcinoma: clinical outcome and safety in elderly patients. $J$ Gastrointestin Liver Dis 2012; 21(4): 397-405.

[28] Nishikawa H, Arimoto A, Wakasa T, Kita R, Kimura T, Osaki Y, Surgical resection for hepatocellular carcinoma: clinical outcomes and safety in elderly patients. Eur J Gastroenterol Hepatol 2013; 25(8): 912-9.

[29] Wong H, Tang YF, Yao TJ, et al. The outcomes and safety of single-agent sorafenib in the treatment of elderly patients with advanced hepatocellular carcinoma (HCC). Oncologist 2011; 16(12): 1721-8.

[30] Di Costanzo GG, Tortora R, De Luca M, et al. Impact of age on toxicity and efficacy of sorafenib-targeted therapy in cirrhotic patients with hepatocellular carcinoma. Med Oncol 2013; 30(1): 446.

[31] Nishikawa H, Kimura T, Kita R, Osaki Y. Treatment for Hepatocellular Carcinoma in Elderly Patients: A Literature Review. J Cancer 2013; 4(8): 635-643.

[32] Morimoto M, Numata K, Kondo M, et al. Higher discontinuation and lower survival rates are likely in elderly Japanese patients with advanced hepatocellular carcinoma receiving sorafenib. Hepatol Res 2011; 41(4): 296-302.

[33] Kudo M, Izumi N, Kokudo N, et al; HCC Expert Panel of Japan Society of Hepatology: Consensus-Based Clinical Practice Guidelines proposed by the Japan Society of Hepatology (JSH) 2010 updated version. Dig Dis 2011; 29: 339-364.

[34] Miller AA, Murry DJ, Owzar K, et al. Phase I and Pharmacokinetic Study of Sorafenib in Patients with Hepatic or Renal Dysfunction. J Clin Oncol 2009; 27: 1800-5.

[35]. McCurry J. Japan battles with obesity. Lancet 2007; 369: 451-52

[36]. Examination Committee of Criteria for 'Obesity Disease' in Japan; Japan Society for the Study of Obesity. New criteria for 'obesity disease' in Japan. Circ J 2002; 66: 987-992.

[37]. Shiwaku K, Anuurad E, Enkhmaa B, Kitajima K, Yamane Y. Appropriate BMI for Asian populations. Lancet 2004; 363: 1077 .

[38] Lencioni R, Llovet JM. Modified RECIST (mRECIST) assessment for hepatocellular carcinoma. Semin Liver Dis 2010; 30(1): 52-60. 
[39] D'Agostino RB Jr: Propensity score methods for bias reduction in the comparison of a treatment to a non-randomized control group. Stat Med 17: 2265-2281, 1998.

[40] Jo M, Yasui K, Kirishima T, et al. Efficacy and safety of sorafenib in very elderly patients aged 80 years and older with advanced hepatocellular carcinoma. Hepatol Res. 2014; [Epub ahead of print].

[41] Iavarone M, Cabibbo G, Piscaglia F, et al; SOFIA (SOraFenib Italian Assessment) study group. Field-practice study of sorafenib therapy for hepatocellular carcinoma: a prospective multicenter study in Italy. Hepatology 2011; 54(6): 2055-63.

[42] Pili R, Guo Y, Chang J, Nakanishi H, Martin GR, Passaniti A. Altered angiogenesis under lying age-dependent changes in tumor growth. J Natl Cancer Inst 1994; 86: 1303-14.

[43] Yao D, Jiang D, Huang Z, et al. Abnormal expression of hepatoma specific gamma-glutamyl transferase and alteration of gamma-glutamyl transferase gene methylation status in patients with hepatocellular carcinoma. Cancer 2000; 88(4): 761-9.

[44] Zhang JB, Chen Y, Zhang B, et al. Prognostic significance of serum gamma-glutamyl transferase in patients with intermediate hepatocellular carcinoma treated with transcatheter arterial chemoembolization. Eur J Gastroenterol Hepatol 2011; 23(9): 787-93.

[45] Shao YY, Hsu CH, Cheng AL. Predictive biomarkers of antiangiogenic therapy for advanced hepatocellular carcinoma: where are we? Liver Cancer. 2013; 2: 93-107.

[46] Sampat KR, O'Neil B. Antiangiogenic therapies for advanced hepatocellular carcinoma. Oncologist. 2013; 18(4): 430-8.

[47] Scartozzi M, Faloppi L, Svegliati Baroni G, et al. VEGF and VEGFR genotyping in the prediction of clinical outcome for HCC patients receiving sorafenib: The ALICE-1 study. Int J Cancer. 2014; [Epub ahead of print].

[48] Lee JH, Chung YH, Kim JA, et al. Genetic predisposition of hand-foot skin reaction after sorafenib therapy in patients with hepatocellular carcinoma. Cancer. 2013; 119(1): 136-42. 